\title{
Recognizing Human Activities User- independently on Smartphones Based on Accelerometer Data
}

\author{
Pekka Siirtola and Juha Röning, Department of Computer Science and Engineering
}

P.O. BOX 4500, FI-90014 University of Oulu, Finland

\begin{abstract}
Real-time human activity recognition on a mobile phone is presented in this article. Unlike in most other studies, not only the data were collected using the accelerometers of a smartphone, but also models were implemented to the phone and the whole classification process (preprocessing, feature extraction and classification) was done on the device. The system is trained using phone orientation independent features to recognize five everyday activities: walking, running, cycling, driving a car and sitting/standing while the phone is in the pocket of the subject's trousers. Two classifiers were compared, $k n n$ ( $k$ nearest neighbors) and QDA (quadratic discriminant analysis). The models for real-time activity recognition were trained offline using a data set collected from eight subjects and these offline results were compared to real-time recognition rates, which are obtained by implementing models to mobile activity recognition application which currently supports two operating systems: Symbian^3 $^{\wedge}$ and Android. The results show that the presented method is light and, therefore, suitable for be used in real-time recognition. In addition, the recognition rates on the smartphones were encouraging, in fact, the recognition accuracies obtained are approximately as high as offline recognition rates. Also, the results show that the method presented is not an operating system dependent.
\end{abstract}

Key words-Activity recognition, classification, mobile phones

\section{INTRODUCTION AND RELATED WORK}

$\mathrm{H}$ UMAN activity recognition using wearable sensors, such as accelerometers, has been widely studied during the recent 20 years. Despite several years of study and promising recognition results, not many commercial products, besides pedometers, exploiting these results are available. There are

Manuscript received June 15, 2012. This work was done as a part of MOPO study (ClinicalTrials.gov Identifier: NCT01376986). Pekka Siirtola would like to thank GETA (The Graduate School in Electronics, Telecommunications and Automation) for financial support. The authors would slso like to thank Infotech Oulu and the Finnish Funding Agency for Technology and Innovation for funding this work.

P. Siirtola is with the University of Oulu, Department of Computer Science and Engineering (e-mail: pekka.siirtola@ee.oulu.fi).

J. Röning is with the University of Oulu, Department of Computer Science and Engineering (e-mail: jjr@ee.oulu.fi). some exceptions, however, such as Polar Active [17] and ActiGraph [1], which can be used to detect the intensity of the activity. Nevertheless, in overall, it seems that companies and people have not been willing to spend money on technology enabling activity recognition. Now, things are about to change: Smartphones are sold more and more every year (smartphone shipments: 2009: 169 million, 2010: 295 million [23]). Smartphones include a wide range of sensors, such as accelerometers, magnetometers, gyroscopes, and GPS, all of which are sensors used for activity recognition in the past studies. Therefore, people already have the technology enabling activity recognition and mobile application stores (AppStore, Nokia Store, Android Market, etc.) can be used to distribute activity recognition applications directly to endusers.

In this article real-time user-independent human activity recognition is presented. The presented method uses only orientation independent features and it is used to recognize five every day activities. Article compares the performance of two classifiers (QDA and $k \mathrm{nn}$ ) in offline and real-time scenarios. Unlike in most of the other studies, in this study the recognition models are implemented to mobile phone to see how models work in real-life, outside laboratory conditions. In addition, models are tested using two different mobile phones: Nokia N8 running Symbian^3 operating system and Samsung Galaxy Mini which is running Android 2.2.1 operating system. It is shown that the method presented in this study enables accurate recognition results not only when the acceleration data is studied offline but also when the whole recognition process (preprocessing, feature extraction and classification) is done in real-time on device. What is more, it is shown that the method is operating system independent.

Human activity recognition using accelerometers has been carried out in various studies, such as [2], [5], [25], [26]. These studies were done using accelerometers build for research use. Therefore, based on these results, it is not straightforward to build a commercial product. There are also some articles where activity recognition using mobile phones has been studied ([3], [14], [16], [19], [24], [27]).

In each of these studies, the data is collected using a mobile phone and the activity recognition is done afterwards on PC, 


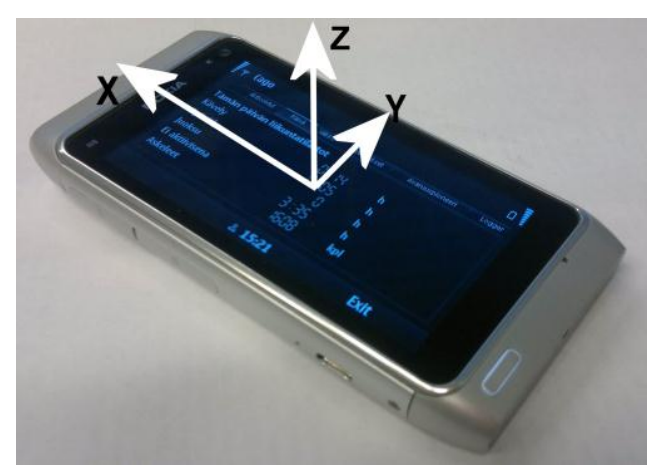

Fig. 1. Nokia N8.

based on collected data. Thus, the activity recognition algorithms are not implemented on the phone, and the classification is not done in real-time on a mobile phone as in our study.

An activity recognition system running purely on a smartphone is presented in [7]. The presented system can be trained on the device and it also does the classification in realtime on the device. The recognition is based on features calculated using geometric template matching and support vector machine (SVM) is used as a classifier. Unfortunately, the article does not include recognition rates: thus, the evaluation of the system is difficult. However, the smartphone application is available from Android Market. The system described in [13] can also be found from Android Market. It seems to recognize activities with high accuracy, but all the features used are not orientation independent. In addition, personalized mobile activity recognition system for Android phones is presented in [8]. In this application user can select which activities he wants application to recognize but it requires training data collection gathered by the user.

Activity recognition using mobile phones has some limitations. Because smartphones are expensive products, people do not want to carry a phone while performing activities where there is a danger to break it. Therefore, it is not necessary to recognize most of the sports activities such as playing football or swimming. Thus, this study concentrates on recognizing five everyday activities, walking, cycling, running, idling (=sitting/standing) and driving/riding a car. The importance of the everyday activity has been shown in several studies, for instance, in [4] it is shown that there is a relationship between moderate intensity lifestyle activity and cardiometabolic health.

Although the latest smartphones are equipped with processors enabling huge calculation capacity, the activity recognition algorithms must nevertheless be light. The mobile phone can be running several applications simultaneously and the activity recognition algorithms are not allowed to use the whole processing power, nor disturb other applications. Therefore, the recognition must be done using light methods.

The paper is organized as follows: Section II describes sensors and data sets. Section III introduces the techniques and methods used in this study. Models trained using offline data and their accuracy are presented in Section IV. Sections V and
V1 evaluates the accuracy of the activity recognition based on models trained using offline data, when detection is done in real-time on a mobile phone running Symbian^3 and Android operation systems. Finally, conclusions are discussed in Section VII.

\section{II.DATA SET}

The data for training the models were collected using a Nokia N8 smartphone [15] running Symbian^3 operating system, Figure 1. N8 includes a wide range of sensors: tri-axis accelerometer and magnetometer, two cameras (12 MP and 0.3 MP), GPS, proximity sensor, compass, microphones and ambient light sensor.

The models used in this study were trained based on activity data collected from eight healthy subjects. The trousers' front pocket was fixed as the phone placement, but the subject was allowed to determine whether the phone was placed in the left or right pocket. The participants performed five different activities: walking, running, cycling, driving a car, and idling, that is, sitting/standing. The total amount of the data collected was about four hours.

These activities were selected because normal everyday life consists mainly of these five activities. Walking and running are different from the other three because everyone has a personal walking and running style. Other activities are not personal, for instance, while cycling, the movement trajectory is predefined. Therefore, the models to recognize walking and running are most challenging to train.

The real-time classification using Nokia N8 was tested by seven subjects, three of whom were subjects whose data were not used to train the recognition models. These subjects carried the phone in their trousers' front pocket and performed from one to five activities.

In addition, the real-time classification was tested using Samsung Galaxy Mini smartphone running Android 2.2.1 operating system. Galaxy Mini is a low budget smartphone having tri-axis accelerometer, proximity sensor, compass and 3.15MP camera. It uses $600 \mathrm{MHz}$ ARMv6 processor. Galaxy Mini was tested by six subjects who carried the phone in their trousers' front pocket and performed from one to five activities.

In this study, only the tri-axis accelerometer was used in this study to detect activities. Accelerometers were running at full speed, which is a phone model dependent feature. However, all the samples were not used in activity recognition process. The latest value from accelerometer was called every 25 milliseconds. Therefore the used sampling frequency was $40 \mathrm{~Hz}$, which is much less than the maximum sampling frequency. The highest possible frequency was not used because it varies between devices and it also varies depending what functions of the phone are used. The used method enables the same sampling frequency to any smartphone, making recognition less phone model dependent.

The training data were collected by subjects whose age 
varied from 25 to 34 years (average 29 years) and height from 1.65 to 1.90 meter (average 1.78 meter) and real-time classification was tested by subjects whose age varied from 27 to 34 years (average 30 years) and height from 1.65 to 1.90 meter (average 1.75 meter). They performed activities outside the laboratory. Subjects walked inside and outside, mainly on flat surface but also in a staircase. Streets where subjects walked, run, drove a car, and cycled were normal tarmac roads, and the route and speed were determined by subjects themselves. Partly the same roads were employed in offline and real-time tests. The roads used for collecting driving a car data included motorways, as well as roads at the city center. Idling consists mostly of office working but includes also standing.

\section{ACTIVITY RECOGNITION}

In this study, two different activity recognition classifiers were compared: quadratic discriminant analysis [10] (QDA) and $k$ nearest neighbors [6] ( $k \mathrm{nn})$. In addition, the recognition was performed using three different settings: (1) offline recognition, to compare different features, classifiers and to evaluate models for online recognition, (2) online recognition on the device, to test the models in realistic real-life conditions, and (3) on device recognition on different phone models to test operating system dependency of the models.

In each case, the raw data were processed in the same way to obtain comparable results.

\section{A. Preprocessing and feature extraction}

The purpose of this study was to develop a user-independent activity recognition method that runs purely on a smartphone and gives accurate recognition results also when the system is used in non-laboratory conditions. The recognition was supposed to work when the mobile phone is placed in the trousers' front pocket. However, the mobile phone can lay on the pocket in numerous different orientations. There are two ways to eliminate the effect of the orientation: (1) by recognizing the orientation of the phone, or (2) by eliminating the orientation information. On the other hand, the orientation is impossible to recognize using only accelerometers. Therefore, the effect of orientation had to be eliminated. In the preprocessing stage, the three acceleration channels were combined as one using square summing to obtain the magnitude acceleration, which is orientation independent. Moreover, the orientation of the phone has limitations, the screen or the back of the phone is always against the user's leg when the phone is in the pocket. Therefore, it was tested if features extracted from a signal where two out of three acceleration channels were square summed would improve the classification accuracy.

The online activity recognition was done using a sliding window technique. The signals from the sensors were divided into equal-sized smaller sequences, also called windows. From these windows, features were extracted and finally the classification of the sequences was done based on these features. In this study, the windows were of the length of 300 observations, which is 7.5 seconds, because the sampling

TABLE I

THE RESULTS OF OFFLINE RECOGNITION USING QDA.

\begin{tabular}{|c|c|c|c|c|c|}
\hline Subject/ Activity & Idling & Walking & Cycling & Driving & Running \\
\hline Idling & $94.3 \%$ & $1.2 \%$ & $0.3 \%$ & $4.2 \%$ & $0.0 \%$ \\
\hline Walking & $1.0 \%$ & $95.6 \%$ & $2.3 \%$ & $0.0 \%$ & $1.3 \%$ \\
\hline Cycling & $0.4 \%$ & $3.4 \%$ & $94.3 \%$ & $1.9 \%$ & $0.0 \%$ \\
\hline Driving & $3.7 \%$ & $0.0 \%$ & $2.2 \%$ & $94.2 \%$ & $0.0 \%$ \\
\hline Running & $0.0 \%$ & $0.0 \%$ & $0.0 \%$ & $0.0 \%$ & $100.0 \%$ \\
\hline
\end{tabular}

TABLE 2

THE RESULTS OF OFFLINE RECOGNITION USING $K$ NN .

\begin{tabular}{|c|c|c|c|c|c|}
\hline Subject/ Activity & Idling & Walking & Cycling & Driving & Running \\
\hline Idling & $94.5 \%$ & $1.1 \%$ & $0.0 \%$ & $4.3 \%$ & $0.0 \%$ \\
\hline Walking & $1.0 \%$ & $90.2 \%$ & $8.6 \%$ & $0.0 \%$ & $0.3 \%$ \\
\hline Cycling & $0.3 \%$ & $1.7 \%$ & $94.6 \%$ & $3.4 \%$ & $0.0 \%$ \\
\hline Driving & $4.2 \%$ & $0.0 \%$ & $2.1 \%$ & $93.8 \%$ & $0.0 \%$ \\
\hline Running & $0.0 \%$ & $0.4 \%$ & $0.0 \%$ & $0.0 \%$ & $99.6 \%$ \\
\hline
\end{tabular}




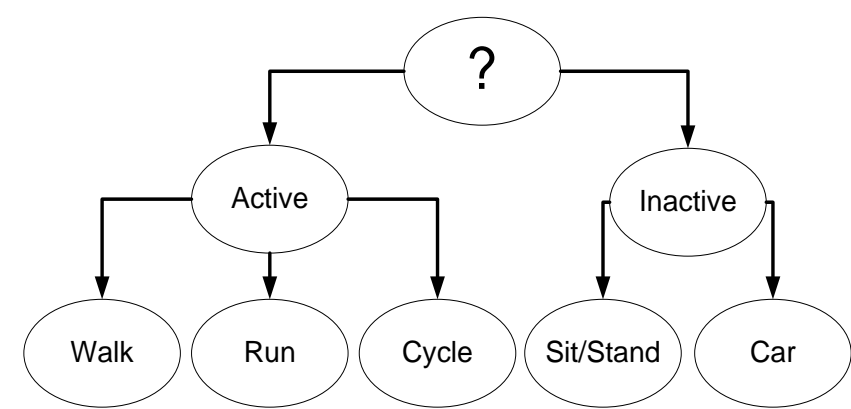

Fig. 2. The decision tree obtained to recognize the type of activity

frequency was $40 \mathrm{~Hz}$. In offline recognition, the slide between two sequential windows was 75 observations, while in online recognition, the slide was set to 150 observations. To reduce the number of misclassified windows, the final classification was done based on the majority voting of the classification results of three adjacent windows. Therefore, when activity changes, a new activity can be detected when two adjacent windows are classified as a new activity. For instance, if the slide is 150 observations, a new activity can be detected after 450 observations, which is around eleven seconds if the sampling rate is $40 \mathrm{~Hz}$.

The total number of 21 features was extracted from magnitude acceleration sequences. These features were standard deviation, mean, minimum, maximum, five different percentiles $(10,25,50,75$, and 90$)$, and a sum and square sum of observations above/below certain percentile $(5,10,25,75$, 90, and 95). The same features were also extracted from the signals where two out three acceleration channels were square summed together. It was noted that the combination of $x$ and $z$ axis signal channels improved the classification most. Therefore, from each window, the total number of 42 orientation independent features were extracted, 21 features from the magnitude acceleration signal and 21 features from the signal where $x$ and $z$ were square summed.

\section{B. Classification}

The classification result was obtained using the decision tree presented in Figure 2, which classifies activities using a two stage procedure. In the first classification stage, a model is trained to decide if the studied subject is currently active (walking, running or cycling) or inactive (driving a car or idling). In the second stage, the exact activity label is obtained. One model has to be trained to classify an active activity as walking, running or cycling, and the other to classify an inactive activity as idling or driving.

The models were trained offline using the collected data. These models were implemented to a smartphones (Symbian^3 and Android) and also used in online tests. To compare different classifiers, the classification was performed using two different classification methods, $k \mathrm{nn}$ and QDA. The most descriptive features for each model were selected using a sequential forward selection (SFS) method [9]. QDA classifiers were trained using the whole training data set, similar to $k \mathrm{nn}$ classifier for the offline recognition. However, because of the limited computational power of the smartphone, the activity recognition on the device was performed using only a limited number of randomly chosen instances from training data.

\section{MODEL TRAINING AND OFFLINE RECOGNITION}

The purpose of the offline recognition is to build and test accurate models that can later be implemented on a mobile phone to enable user-independent and operating system independent real-time recognition of the activities on the device. Models were trained for $k \mathrm{nn}$ and QDA classifiers based on the data collected from eight persons.

\section{A. Results}

To obtain reliable user-independent results, the training was performed using the leave-one-out method, so that each person's data in turn was used for testing and the other seven sequences were employed for model training.

The results are shown in Tables 1 and 2.

\section{B. Classification}

The offline recognition results show that the both classifiers, QDA and $k \mathrm{nn}$, enable accurate results. The average classification accuracy using QDA is $95.4 \%$, while $k \mathrm{nn}$ enables

TABLE 3

THE RESULTS OF ONLINE RECOGNITION ON DEVICE USING NOKIA N8 AND KNN

\begin{tabular}{|c|c|c|c|c|c|c|}
\hline $\begin{array}{c}\text { SUBJECT/ } \\
\text { ACTIVITY }\end{array}$ & IDLING & WALKING & CYCLING & DRIVING & RUNNING & AVERAGE \\
\hline SUBJECT 1 & $91.5 \%$ & $99.9 \%$ & $89.2 \%$ & $91.4 \%$ & $87.2 \%$ & $91.8 \%$ \\
\hline SUBJECT 2 & $99.9 \%$ & $99.9 \%$ & $93.7 \%$ & $87.8 \%$ & $92.4 \%$ & $94.7 \%$ \\
\hline SUBJECT 3 & $76.3 \%$ & $99.9 \%$ & $89.6 \%$ & - & $97 \%$ \\
\hline SUBJECT 4 & - & - & - & $97.6 \%$ & $97.9 \%$ & $94.6 \%$ \\
\hline SUBJECT 5 & $95.6 \%$ & $99.9 \%$ & $89.5 \%$ & $89.4 \%$ & $99.9 \%$ & $96.9 \%$ \\
\hline SUBJECT 6 & $94.1 \%$ & $99.9 \%$ & $93.8 \%$ & - & $99.9 \%$ & $99.4 \%$ \\
\hline SUBJECT 7 & $83.3 \%$ & $99.8 \%$ & $98.1 \%$ & - & 99 \\
\hline
\end{tabular}


TABLE 4

THE RESULTS OF ONLINE RECOGNITION ON DEVICE USING NOKIA N8 AND QDA.

\begin{tabular}{|c|c|c|c|c|c|c|}
\hline $\begin{array}{l}\text { SUBJECT/ } \\
\text { ACTIVITY }\end{array}$ & IDLING & WALKING & CYCLING & DRIVING & RUNNING & AVERAGE \\
\hline SUBJECT 1 & $98.5 \%$ & $65.6 \%$ & $95.7 \%$ & $99.6 \%$ & $91.5 \%$ & $90.2 \%$ \\
\hline SUBJECT 2 & $99.9 \%$ & $97.6 \%$ & $91.2 \%$ & $88.5 \%$ & $99.2 \%$ & $95.3 \%$ \\
\hline SUBJECT 3 & $99.9 \%$ & $97.6 \%$ & $91.3 \%$ & - & $99.8 \%$ & $96.9 \%$ \\
\hline SUBJECT 4 & - & - & - & $87.2 \%$ & - & $87.2 \%$ \\
\hline SUBJECT 5 & $98.2 \%$ & $99.3 \%$ & $97.6 \%$ & $98.1 \%$ & $99.9 \%$ & $98.6 \%$ \\
\hline SUBJECT 6 & $99.9 \%$ & $96.0 \%$ & $93.8 \%$ & - & $99.9 \%$ & $97.4 \%$ \\
\hline SUBJECT 7 & $99.9 \%$ & $99.8 \%$ & $98.1 \%$ & - & $99.9 \%$ & $99.4 \%$ \\
\hline
\end{tabular}

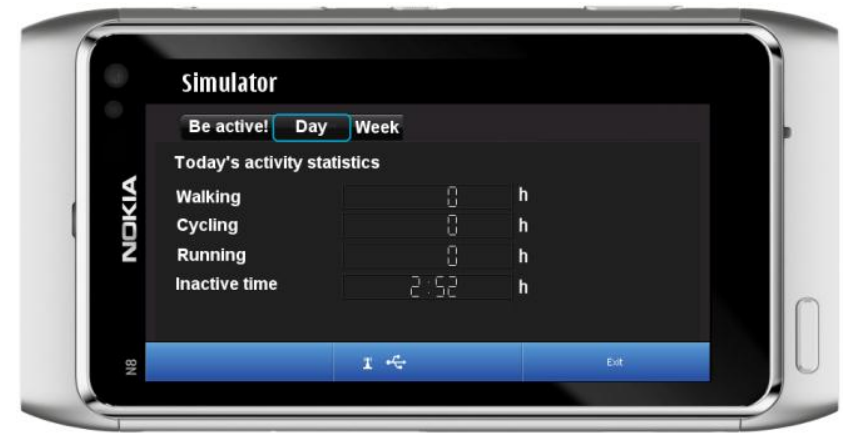

Fig. 3. Activity recognition application for Symbian^3 smartphones.

an accuracy of $94.5 \%$. It should be noted, however that this difference is not statistically significant according to paired $t$ test. Also, each of the five activities are recognized with high accuracy.

\section{V.REAL-TIME EXPERIMENTS ON DEVICE USING NOKIA N8}

An activity recognition application for Symbian^3 devices was build using Qt [18] programming language, Figure 3. Every Nokia phone running a Symbian^ 3 operating system has the same kind of accelerometers, and therefore, the results presented in this section can be obtained using any Nokia Symbian^$^{\wedge} 3$ phone. The application uses the activity recognition models that were trained using the data presented in Section II. It should be noted that offline recognition employing $k \mathrm{nn}$ uses the whole training data set to recognize activities from the test data, making the classification process complex. As mentioned before, because of the limited computational power of the smartphone, the recognition on the device was performed using only a limited number of randomly chosen instances from training data. In this study, eight instances from each activity per subject were chosen as instances of $k \mathrm{nn}$-based recognition model. QDA -based realtime classification results were obtained using the very same models used in offline recognition.

\section{A. Results}

The application and the real-time classification were tested by seven persons carrying Nokia N8 smartphone on their trousers' front pocket. Three of these were different from the eight subjects that collected the data for training the recognition models. The recognition results are shown in Tables 3 and 4. Both classifiers were running on the device in parallel; thus, the results are comparable.

\section{B. Discussion}

The real-time experiment showed that the application and models are running smoothly on the device. When activity recognition is done using QDA classifier, the application uses under 5\% of the CPU's (680Mhz ARM11 processor) capacity. Therefore, the application can be employed alongside other applications, such as games. The usage of $k \mathrm{nn}$ as a classifier uses slightly more CPU capacity. In addition, the recognition rates on the device are around as high as offline. The average recognition rate using QDA is $95.8 \%$, while using $k \mathrm{nn}$ it is slightly lower, 93.9\%. According to paired $t$-test, this difference is not statistically significant, however.

Online recognition was tested by subjects (subjects 1, 2 and 3 ) whose data was not used for training as well as subjects (subjects 4, 5, 6 and 7) whose data was used for training. In both cases, the average recognition rate is high. However, there are two cases where user-independent classification has not succeeded very well. Walking activity of Subject 1 was recognized only with the rate of $65.6 \%$ when QDA is used as a classifier and cycling of Subject 3 using $k \mathrm{nn}$ was recognized correctly only in $76.3 \%$ of the cases. In both cases, cycling and walking were mixed together. It seems that inner class variation of these activities is too low causing misclassification. As mentioned above, walking is one of the most difficult activities to recognize user-independently, because every subject has a personal walking style. In addition, not the whole training data were used to train the $k \mathrm{nn}$ model to keep to recognition process light, which may have caused the weak recognition rates with Subject 3's cycling activity. In overall, the recognition on the device works well, 
however. It seems that the first phase of the recognition, where observations are classified as active or inactive, appears to work almost perfectly. Therefore, to make recognition even more accurate, the second phase of the classification should be improved.

The models used in online recognition are user-independent and it also seems that they are "car-independent". Two different cars were used in the data collecting phase. Although during the online test, subjects 1 and 2 used a car not used to train the models, the recognition rate is still high. On the other hand, to make sure that the models are car-independent, more tests should be carried out using different cars and road conditions.

\section{REAL-TIME EXPERIMENTS ON DEVICE USING ANDROID- PHONE}

According to the results of the previous section, both tested classifiers produce as good results. However, QDA is simpler than $k \mathrm{nn}$ and, therefore, it is lighter and more suitable for be used in an application that is supposed to run at the background all the time. Android-version of the activity recognition application was built after Symbian^3-version using Java programming language. Therefore, based on the experiences gathered using Nokia phones, it was decided that Android-version would use QDA as a classifier.

\section{A. Results}

Real-time classification on Android device was tested by five subjects, again, carrying the phone on their trousers' front pocket. Subject 1's data was not used in to train the models. The data of other five subjects were used to train models but data is different to the one used in this section. The results are shown in Table 5. The purpose of these experiments was to show that the presented activity recognition method is operating system independent.

\section{B. Discussion}

The results show that activity recognition models are accurate also when they are running in Android-based smartphone. Therefore, the presented method is not an operating system dependent.

Noticeable is the weak recognition accuracy of the activity driving a car. Only $67.8 \%$ of the cases were recognized correctly when Subject 3 was using the application. However, Subject 3 had to stop several times during the data collection session because of the red traffic lights, and in this study, these stops were considered as driving a car. If these stops are considered as idling and therefore removed from driving results, the recognition rate of driving would be almost $100.0 \%$. On the other hand, in the case of Subject 4, driving was recognized with a rate of $98.8 \%$. This subject did not have to wait at traffic lights.

However, driving was the only activity recognized with a low rate. All the other activities were recognized with really high accuracy and the average recognition rate is $96.5 \%$. For instance, running is recognized perfectly with a rate of $100.0 \%$. Moreover, the recognition accuracy of walking is also nearly $100 \%$. The cycling did mix up with walking a little but still the average recognition rate of cycling was as high as $94.5 \%$. In fact, the average recognition rate on a device using Samsung Galaxy Mini is higher than offline recognition rate and recognition rate using Nokia N8. However, these results are not fully comparable because they are based on separate data collections.

\section{CONCLUSIONS}

Orientation independent real-time activity recognition of five everyday activities using a mobile phone was introduced in this study. The whole classification process, including preprocessing, feature extraction, and classification, was done on the device. Recognition accuracies were tested using two classifiers ( $k n n$ and QDA) to compare different classification algorithms. In addition, real-time activity recognition using QDA as a classifier was tested using two different phones (Nokia N8 and Samsung Galaxy Mini) running different operating systems (Symbian^3 and Android). These operating systems were chosen to this study, because not only they are popular, but also they enable real multitasking, unlike some other smartphone operating systems, such as iOS and Windows Phone 7. User-independent models for online recognition were trained offline using a data set collected by eight subjects.

When the recognition rates of on device recognition using Nokia N8 are studied in detail it can be seen that using the models trained offline, the recognition rates on Nokia N8 device are around as high as offline recognition results. In the offline case, the average classification accuracy based on the

TABLE 5

THE RESULTS OF ONLINE RECOGNITION ON DEVICE USING SAMSUNG GALAXY MINI AND QDA.

\begin{tabular}{|c|c|c|c|c|c|c|}
\hline $\begin{array}{c}\text { SUBJECT/ } \\
\text { ACTIVITY }\end{array}$ & IDLING & WALKING & CYCLING & DRIVING & RUNNING & AVERAGE \\
\hline SUBJECT 1 & $92.5 \%$ & $97.1 \%$ & $100.0 \%$ & -- & $100.0 \%$ & $97,4 \%$ \\
\hline SUBJECT 2 & $91.7 \%$ & $100.0 \%$ & $98.9 \%$ & -- & $100.0 \%$ & $97.7 \%$ \\
\hline SUBJECT 3 & $100.0 \%$ & $100.0 \%$ & $90.9 \%$ & $67.8 \%$ & -- & $91.7 \%$ \\
\hline SUBJECT 4 & -- & - & -- & $98.8 \%$ & $100.0 \%$ & $98.8 \%$ \\
\hline SUBJECT 5 & $96,7 \%$ & $97.8 \%$ & $87.5 \%$ & -- & -- & $97.9 \%$ \\
\hline SUBJECT 6 & $98.5 \%$ & $100.0 \%$ & $95.2 \%$ & -- & & 97.9 \\
\hline
\end{tabular}


data used to train the models using QDA is $95.4 \%$, while $k \mathrm{nn}$ enables an accuracy of $94.5 \%$. While performing online recognition on Nokia $\mathrm{N} 8$, the average recognition rate using QDA is $95.8 \%$, while using $k \mathrm{nn}$ it is slightly lower, $93.9 \%$. However, in some cases, user-independent real-time recognition results on Nokia N8 are not as high as expected. In order to achieve even more accurate online results with every subject, the training data should contain more variation. Now it seems that in some cases the models for online recognition are build using too homogeneous a data set, and therefore, the models are not as good as they could be. Nevertheless, the results are encouraging. Moreover, activity recognition application runs smoothly on N8. It uses under $5 \%$ of CPU capacity when QDA is employed as a classifier; thus, other applications can be run alongside. Using $k n n$ as a classifier requires more CPU capacity.

Because of the accuracy and lightness of the QDA classifier based activity recognition, Android-version of the application was tested only using QDA. Android-version was tested by six subjects carrying Samsung Galaxy Mini smartphone on their trousers' pocket. The results are really good with every subject. For instance, running is recognized perfectly with rate $100.0 \%$. All the other activities were also recognized with really high accuracy. The only exception was driving a car activity, where detection accuracy was lower than expected with one test subject. However, the main reason for this was red traffic lights which caused long unwanted stops.

Based on the results it is clear that activity recognition works reliably operating system independently. It seems that in this study, the on device recognition results using Androidphone are a little higher than the ones gained using Symbian^3 phone. However, subjects did not carry Symbian^ 3 and Android phones at the same time, the real-time recognition results of Tables 4 and 5 are not fully comparable.

Real-time activity recognition on device is working reliably on both tested operating systems, Symbian^ 3 and Android, though there are differences in accelerometers between phone models and operating systems. The main difference of accelerometers is the maximum sampling rate. However, in this study, the maximum sampling rate was not used. Though, the accelerometers were running at full speed, but still, a new value to be used in activity recognition was called every 25 millisecond. Therefore, the used frequency was $40 \mathrm{~Hz}$, which is much less than the maximum frequency of most of the smartphones. Thus, the presented method can be used with every smartphone and it is not dependent on the phone model.

Real-time recognition on the device was only tested by predefined five activities and not when the subject is doing something else. Null-data recognition is not included in this study, and therefore, such activities cause incorrect classifications. Thus, to improve the accuracy of the application, null-activity recognition should be included. Also building a behavior recognition system based on the activity recognition results could reduce the number of misclassifications [12]. In addition, it should be tested how different trousers affect the results, now every test subject was wearing jeans.

The presented activity recognition application is not body position independent. The system is trained to recognize activities when the phone is placed to the subject's trousers' pocket. Although trousers' pockets are the most common place to carry a phone [11], especially among males, a body position independent approach should be considered. Body position independent recognition is naturally more difficult than position dependent, and therefore, most likely the recognition rates would not be as high as the ones presented in this study. However, high position independent recognition rates are for instance achieved in [14].

Although, the recognition accuracy on the device is excellent, there are still some remaining issues. The application uses too much battery and, therefore, even lighter methods should be used. For instance, human activities can be recognized from lower frequency signals than the ones used in this study [21]. Therefore, the sampling frequency could be reduced or the number of required classifications could be reduced by using periodic quick-test [22]. However, even now without memory and processing power optimization, the battery of Nokia N8 and Samsung Galaxy Mini lasts over 24 hours while the application is running at the background.

In this study, everything except model training is done on the device. Other option would be to send the accelerometer data to the server, perform the classification process there and send the results to a mobile phone. In this case, calculation capacity would not be an issue, but on the other hand, privacy issues should be handled. Moreover, data transfer is not free and can cause exceptionally high costs, especially when the mobile phone and application are used abroad.

\section{ACKNOWLEDGMENT}

The authors would thanks to Tero Vallius for Qt coding and all the partners of MOPO-study (ClinicalTrials.gov Identifier: NCT01376986).

\section{REFERENCES}

[1] ActiGraph: http://www.theactigraph.com/

[2] Bao, L., Intille, S.S.: Activity recognition from user-annotated acceleration data. Pervasive 2004 pp. 1--17 (2004).

[3] Brezmes, T., Gorricho, J.L., Cotrina, J.: Activity recognition from accelerometer data on a mobile phone. In: Distributed Computing, Artificial Intelligence, Bioinformatics, Soft Computing, and Ambient Assisted Living, Lecture Notes in Computer Science, vol. 5518, pp. 796--799. Springer Berlin / Heidelberg (2009)

[4] Camhi, S.M., Sisson, S.B., Johnson, W.D, Katzmarzyk, P.T., TudorLocke, C., Accelerometer-determined moderate intensity lifestyle activity and cardiometabolic health, Preventive Medicine, Volume 52, Issue 5, 1 May 2011, Pages 358-360

[5] Ermes, M., Pärkkä, J., Mäntyjärvi, J., Korhonen, I.: Detection of daily activities and sports with wearable sensors in controlled and uncontrolled conditions. Information Technology in Biomedicine, IEEE Transactions on 12(1), 20--26 (Jan. 2008)

[6] Fix, E., Hodges, J.L.: Discriminatory analysis: Nonparametric discrimination: Consistency properties. Tech. Rep. Project 21-49-004, Report Number 4, USAF School of Aviation Medicine, Randolf Field, Texas (1951) 
[7] Frank, J., Mannor, S., Precup, D.: Activity recognition with mobile phones. In: Machine Learning and Knowledge Discovery in Databases, Lecture Notes in Computer Science\}, vol. 6913, pp. 630--633. Springer Berlin / Heidelberg (2011)

[8] Gomes, J., Krishnaswamy, S., Gaber, Mohamed, Sousa, P. and Menasalvas, E. (2012) MARS: a personalised Mobile Activity Recognition System. In: Proceedings of the IEEE International Conference on Mobile Data Management, 23-26 July, 2012, Bengaluru, India.

[9] Haapalainen, E., Laurinen, P., Junno, H., Tuovinen, L., Röning, J.: Feature selection for identification of spot welding processes. Proceedings of the 3rd International Conference on Informatics in Control, Automation and Robotics pp. 40--46 (2006)

[10] Hand, D.J., Mannila, H., Smyth, P.: Principles of data mining. MIT Press, Cambridge, MA, USA (2001)

[11] Ichikawa, F., Chipchase, J., Grignani, R.: Where's the phone? a study of mobile phone location in public spaces. In: Mobile Technology, Applications and Systems, $20052^{\text {nd }}$ International Conference on, pp. 1 $-8(2005)$

[12] Koskimäki H, Huikari V, Siirtola P \& Röning J : Behavior modelling in industrial assembly lines using a wrist-worn inertial measurement unit, accepted to Journal of Ambient Intelligence and Humanized Computing (2012)

[13] Kwapisz, J.R., Weiss, G.M., Moore, S.A.: Activity recognition using cell phone accelerometers. SIGKDD Explor. Newsl. 12, 74--82 (2011)

[14] Lu, H., Yang, J., Liu, Z., Lane, N.D., Choudhury, T., Campbell, A.T.: The Jigsaw continuous sensing engine for mobile phone applications. In: Proceedings of the 8th ACM Conference on Embedded Networked Sensor Systems, SenSys '10, pp. 71-84 (2010)

[15] Nokia N8: http://europe.nokia.com/find-products/devices/nokia-n8

[16] Peebles, D., Lu, H., Lane, N.D., Choudhury, T., Campbell, A.T.: Community-guided learning: Exploiting mobile sensor users to model human behavior. In: Proceedings of the Twenty-Fourth AAAI Conference on Artificial Intelligence, AAAI 2010, Atlanta, Georgia, USA, July 11-15 (2010)

[17] Polar 451

[18] Qt: http://qt.nokia.com/

[19] Ryder, J., Longstaff, B., Reddy, S., Estrin, D.: Ambulation: A tool for monitoring mobility patterns over time using mobile phones. In: Computational Science and Engineering, 2009. CSE '09. International Conference on, vol. 4, pp. 927 --931 (2009)

[20] Samsung Galaxy http://www.samsung.com/hk_en/consumer/mobile/mobilephones/smartphone/GT-S5570EGAVDC-spec

[21] Siirtola P, Laurinen P, Röning J \& Kinnunen H (2011): Efficient Accelerometer-Based Swimming Exercise Tracking, IEEE Symposium on Computational Intelligence and Data Mining (CIDM 2011), April 11-15, 156--161.

[22] Siirtola, P., Koskimäki, H., Röning, J.: Periodic quick test for classifying long-term activities. In: IEEE Symposium on Computational Intelligence and Data Mining (CIDM 2011), pp. 135--140 (2011)

[23] Smartphone shipments: http://www.bgr.com/2011/03/10/bergsmartphone-shipments-grew-74-in-2010/

[24] Sun, L., Zhang, D., Li, B., Guo, B., Li, S.: Activity recognition on an accelerometer embedded mobile phone with varying positions and orientations. In: Ubiquitous Intelligence and Computing, Lecture Notes in Computer Science, vol. 6406, pp. 548--562. Springer Berlin / Heidelberg (2010)

[25] Suutala, J., Pirttikangas, S., Röning, J.: Discriminative temporal smoothing for activity recognition from wearable sensors. In: Proc. of the 4th International Symposium on Ubiquitous Computing Systems (UCS07), pp. 182--195. Tokyo, Japan (2007)

[26] Van Laerhoven, K., Cakmakci, O.: What shall we teach our pants? Wearable Computers, 2000. The Fourth International Symposium on pp. $77-83$ (2000)

[27] Wang, S., Chen, C., Ma, J.: Accelerometer based transportation mode recognitionnon mobile phones.Wearable Computing Systems, AsiaPacific Conference on, 44--46 (2010)
P. Siirtola received his M.Sc. degree in Mathematics from University of Oulu, Oulu, Finland, in 2006. At the moment he does graduate studies in Department of Computer Science and Engineering where he works as a research scientist, sponsored by the Graduate School in Electronics, Telecommunications and Automation (GETA). His research interests include pattern recognition, data mining and machine learning methods and applications.

J. Röning obtained the degree of Doctor of Technology in 1992, Licentiate in Technology with honors in 1985, and Diploma in Engineering (MSEE) with honors in 1983, all at the University of Oulu in Finland. From 1983 he has been a member of faculty of the University of Oulu, where he is currently Professor of Embedded System and head of the Department of Computer Science and Engineering. Professor Röning has two patents and has published more than 200 papers. His main research interests are in intelligent systems, especially mobile robots, machine vision, and software security. 